\title{
La propiedad familiar como mecanismo de gobierno disciplinador de la dirección en las empresas mexicanas Una evidencia empírica
}

\author{
Luis Ángel Castrillo Lara* \\ Juan Manuel San Martín Reyna**
}

\section{Resumen}

El presente trabajo analiza la interacción entre los mecanismos de gobierno de la empresa (estructura de propiedad, consejo de administración y deuda) centrándonos especialmente en el estudio de la estructura de propiedad, en concreto el control familiar, como mecanismo de supervisión y control de la dirección. Tras comprobar que la propiedad familiar constituye un elemento disciplinador del equipo directivo, se analiza el efecto de los mecanismos de gobierno sobre la discrecionalidad directiva segmentando la muestra por estructura de propiedad - familiares y no familiares-; del análisis efectuado se observa que mecanismos de control que resultaban relevantes dejan de serlo debido a que uno de ellos, concretamente la estructura de propiedad familiar, alivia los problemas de agencia evitando incurrir en mecanismos de control adicionales como la deuda. Para el análisis de los datos se parte de un estudio de corte transversal, donde se llevan a cabo dos modelos, uno al que denominamos global en el que se realiza el estudio considerando la muestra de forma conjunta, y otro al que denominamos individual en el que se lleva a cabo el análisis dividiendo la muestra en empresas familiares y no familiares. Para ello, hemos utilizado un conjunto de empresas que han cotizado en la Bolsa Mexicana de Valores durante los años 2003 y 2004. Como variable representativa de la discrecionalidad directiva hemos tomado los ajustes por devengo discrecionales obtenidos de la aplicación del modelo de Jones en su versión de corte transversal.

Palabras clave: mecanismos de gobierno, ajustes por devengo, empresa familiar

Fecha de recepción: 23-03-2006

Fecha de aceptación: 11-01-2007

The Ownership Family Like Control Mechanism of The Direction in The Mexican Companies: An Empirical Evidence

\begin{abstract}
The present work analyzes the interaction among the mechanisms of corporate governance -ownership structure, board composition and leverage-; focusing in the study

\footnotetext{
* Profesor de la Universidad de Burgos. Correo electrónico: 1castri@ubu.es

**Profesor investigador de la Universidad Autónoma de Tamaulipas. Correo electrónico: jsanmartin@uat.edu.mx
}

No. 222, mayo-agosto 2007:59-82 
of the property structure, in particular, the family control, like mechanism of supervision and control of the direction. After verifying that the ownership family constraining the managers manipulation accruals, the effect of the control mechanisms is analyzed on the discretionary accruals separating the sample by ownership structure - family and nonfamily-, finding that control mechanisms which were important, stopped being because one of them, specially the structure of family ownership, alleviates to the agency problems avoiding incurring additional control mechanisms like the debt. For the analysis of the data we left from a study of cross section with two models, one that we denominated global where the study is made considering the sample of joint form, and another one to which we denominated individual, in which we made the analysis dividing the sample in family and nonfamily firms. For it, we have used a set of companies that have quoted in Mexican stock market during 2003 and 2004. As variable representative of managers manipulation we have taken the discretionary accruals obtained from the application of the Model of Jones is its version of cross section.

Keywords: Mechanisms of governance, Discretionary accruals, Family firms.

\section{Introducción}

$\mathrm{H}^{2}$ asta no hace mucho tiempo, la mayoría de los trabajos de investigación sobre gobierno corporativo se habían centrado en el análisis de algunos mecanismos de gobierno, en particular los relacionados con la estructura de propiedad y consejo de administración, limitándose, casi siempre, a analizar el efecto unilateral de los mecanismos, presuponiendo que entre sí éstos son independientes. Recientes estudios, como por ejemplo los de Kini et al. (1995), Agrawal y Knoeber (1996), Coles et al. (2001) y Bushman y Smith (2001), parecen situarnos en una nueva generación de investigación en gobierno corporativo, en la cual se reconoce de forma explícita la interacción entre los mecanismos de gobierno. En esta nueva línea, se trata de profundizar en la posible interacción entre dichos mecanismos poniendo de manifiesto la capacidad de la empresa para diseñar un sistema de gobierno corporativo eficiente a través de la selección de diferentes mecanismos, que se sustituyen o complementan.

Dado este efecto de sustitución o complementariedad de los mecanismos de gobierno, derivado de la similitud en su aplicación, pueden usarse diferentes alternativas para controlar los problemas de agencia en la empresa de tal manera que el uso de cada uno de los mecanismos depende del uso que se haga de los demás, por lo que pierde importancia el efecto individual de cada uno de ellos sobre la creación de valor de la empresa en relación con el efecto conjunto de todos los mecanismos elegidos (Argrawal y Knoeber, 1996). Es en este punto precisamente donde encontramos la justificación al trabajo que aquí presentamos; además, tratamos de analizar el efecto de diferentes mecanismos de gobierno como estructura de propiedad, consejo de administración y endeudamiento en la discrecionalidad directiva, atendiendo al hecho de que estos dos últimos pueden tener diferentes impactos, lo que depende de la estructura de propiedad de la empresa. 
La propiedad familiar como mecanismo de gobierno disciplinador de la

dirección en las empresas mexicanas: una evidencia empírica

El hecho de que se contemplen estos mecanismos dependientes de la estructura de propiedad proviene de la consideración de ésta como endógena; es decir, la estructura de propiedad será aquella que resulte más apropiada teniendo en cuenta las características del entorno en el que se desenvuelve la compañía (Shlelifer y Vishny, 1997; La Porta et al., 2000), por lo que los mecanismos de gobierno podrían variar dependiendo de la estructura de propiedad de cada empresa.

Desde esta perspectiva, el objetivo de nuestro trabajo consiste en analizar la relación entre los mecanismos de gobierno - estructura de propiedad, consejo de administración y deuda - y la discrecionalidad directiva, haciendo especial hincapié en el estudio de la estructura de propiedad, en concreto, el control familiar, como mecanismo disciplinador del equipo directivo. Después, segmentamos la muestra por estructura de propiedad, es decir, en empresas familiares y no familiares para escudriñar la idea de que la concertación de propiedad hará que mecanismos de control que resultan significativos cuando analizamos las empresas de manera conjunta dejen de serlo al dividir la muestra debido a que uno de los mecanismos, específicamente la estructura de propiedad familiar, solventa los problemas de agencia evitando incurrir en otros costes que implican mecanismos de control adicionales, como la deuda. Para ello, hemos tomado una muestra de empresas que han cotizado en la Bolsa Mexicana de Valores durante los años 2003 y 2004. Como variable representativa de la discrecionalidad directiva hemos tomado los ajustes por devengo discrecionales obtenidos de la aplicación del modelo de Jones en su versión de corte transversal.

Para conseguir los objetivos propuestos, tras este apartado introductorio, dedicaremos el segundo epígrafe a los fundamentos teóricos, abordando, desde una óptica general, el estudio de la teoría de la agencia y del concepto de gobierno corporativo, profundizando especialmente en el estudio de los mecanismos de control interno y la empresa familiar, así como en sus efectos sobre la discrecionalidad directiva. Posteriormente, en el tercer epígrafe se plantean las hipótesis y la metodología empleada en el análisis empírico. Después, se presenta los resultados obtenidos en el cuarto epígrafe. Finalmente, el quinto epígrafe presenta las conclusiones del trabajo.

\section{El gobierno de la empresa: fundamentos teóricos}

El debate sobre la influencia de los mecanismos de gobierno sobre la discrecionalidad directiva debe ser situado en el contexto del problema de agencia derivado de la separación entre propiedad y control de la empresa, lo que genera la aparición de asimetrías entre los intereses de directivos y accionistas (Jensen y Meckling, 1976). Cuando los directivos no son propietarios de la compañía que dirigen, su comportamiento se ve afectado por intereses propios que alejan sus objetivos de la maximización del valor de la empresa y, en consecuencia, de los intereses de los accionistas o propietarios (Berle y Means, 1932; Jensen y Meckling, 1976; Fama, 1980; Fama y Jensen, 1983). En este sentido, la separación de propiedad y control, tal y como se presenta en la empresa moderna, tiene como principal problema evitar los posibles comportamientos oportunistas de los gestores que tienden 
a reducir el valor de la compañía (Gedajlovic y Shapiro, 1998). Ante esta situación, la bibliografía sobre gobierno corporativo ha hecho hincapié en los problemas que plantea la relación entre accionistas y directivos, estudiando los mecanismos de que disponen los inversores para ejercer control sobre la compañía de tal forma que los problemas se vean minimizados (Shleifer y Vishny, 1997). Estos mecanismos se clasifican según su carácter en externos o internos a la organización.

Ahora bien, la forma en que se ha articulado la propiedad y control registra diferencias entre países. Es posible distinguir entre sistemas financieros en los que el gobierno de la empresa descansa en mecanismos internos, como pueden ser el consejo de administración o la estructura de propiedad, y aquellos en los que predomina el carácter externo, en última instancia, la propia disciplina del mercado (Fernández y Gómez-Ansón, 1999). En este sentido, la determinación del tipo de gobierno y, por ende, los mecanismos por aplicar en cada caso dependen en gran medida de las características institucionales y corporativas en las que se encuentre enmarcada la empresa. Bajo este escenario, la estructura de propiedad podría considerarse como endógena; es decir, será aquella que resulte más apropiada considerando las características de la compañía y el entorno en el que se desenvuelve (Shlelifer y Vishny, 1997; La Porta et al., 2000) de tal forma que los mecanismos de gobierno tenderán a establecerse de acuerdo con el país, ya que existen elementos de su propio entorno que influirán en la configuración de la forma de gobierno de las empresas.

\subsection{Mecanismos de gobierno como factor de control de la discrecionalidad directiva}

Desde una perspectiva financiera, es decir, circunscribiendo el problema de gobierno y sus mecanismos a la relación entre accionistas y directivos, el planteamiento que subyace bajo esta relación es que una mayor eficiencia en la supervisión y el control de la dirección, llevada a cabo a través de diferentes mecanismos de gobierno, supondrán una reducción en los costes de agencia que, en definitiva, redundarán en un mayor valor de la empresa (Azofra y Santamaría, 2002).

Estos mecanismos han sido analizados por un gran número de investigadores. La clasificación más aceptada distingue dos tipos: un mecanismo de control externo, con predominio de mecanismos basados en las actuaciones de los mercados, frente a otra categoría denominada de control interno, en la que los incentivos descansan fundamentalmente en elementos como estructura de propiedad, consejo de administración y endeudamiento (Fernández y Gómez-Ansón, 1999).

\subsubsection{Estructura de propiedad}

De acuerdo con Salas (2002), el análisis de la estructura de propiedad de la empresa puede llevarse a cabo a través del estudio de tres elementos principales: a) la naturaleza del grupo que controla la empresa (familias), b) el grado de concentración del accionariado 
La propiedad familiar como mecanismo de gobierno disciplinador de la

dirección en las empresas mexicanas: una evidencia empírica

(porcentaje de acciones que directa o indirectamente poseen los accionistas mayoritarios) y c) la participación accionarial de los internos.

En cuanto a la naturaleza del grupo controlador, la identidad de los grandes accionistas tiene importantes implicaciones en el gobierno de la empresa. Sin embargo, es necesario considerar que no todos los grandes inversores poseen los mismos incentivos para supervisar a los gestores. La concentración de la propiedad y su capacidad de supervisión está más relacionada con la característica de los propietarios de la empresa que con la propia empresa (Fernández y Gómez-Ansón, 1999). Así, grandes accionistas, como lo son las familias, poseen determinadas características en su actuación que hacen especialmente relevante el estudio de su papel como mecanismo supervisor.

Cuesta (2000) señala que son dos los requisitos que permiten definir la empresa familiar. El primero es de carácter objetivo y consiste en que la propiedad de la empresa - o al menos una parte significativa - esté concentrada en una sola persona o en un número reducido de personas ligadas por un vínculo de sangre que intervienen de forma importante en la gestión de la empresa. El segundo requisito es subjetivo y consiste en la voluntad de que este estado de cosas perdure en el tiempo; por tanto, la propiedad y el control de la empresa se transmitirá a los sucesores miembros de la familia. En este sentido, autores como Casson (1999) y Chami (1999) argumentan que las empresas familiares ven a la compañía como un activo para legar a los miembros de la familia o a sus descendientes y no como riqueza por consumir durante su curso de vida. Así, pues, la supervivencia de la compañía es una preocupación importante para las familias, lo cual sugiere que éstas tienen una mayor probabilidad de maximizar el valor de la empresa.

Una característica común de las empresas familiares es que los miembros de la familia sirvan como directivos de éstas o desempeñen a menudo otras labores de la alta dirección. Autores como Jensen y Meckling (1976) y Fama y Jensen (1983) argumentan que el coste de reducir asimetrías informativas y problemas de agencia es menor cuando los propietarios de la empresa participan de forma activa en la dirección de ésta. Así, bajo el enfoque de la teoría de la agencia, se podría argumentar que la implicación familiar en la propiedad y dirección del negocio proporciona a la empresa familiar ciertas ventajas en la medida en que, según este enfoque teórico, una empresa donde coincidieran propietarios y directivos debería ser más eficiente que aquellas en las que no se da esta identificación dados los problemas del posible comportamiento oportunista del agente con respecto al principal y los costes asociados a la supervisión del mismo (Cabrera et al. 2000). De esta forma, los grandes accionistas o controladores son capaces de limitar los problemas de agencia inherentes a la separación entre propiedad y control, ya que estos accionistas tendrán una menor probabilidad de permitir que gerentes profesionales actúen de manera oportunista (Shleifer y Vishny, 1986). Planteado de esta forma, la propiedad familiar permite una convergencia de intereses entre dirección y accionistas, lo que lleva hacia una maximización del valor de la empresa. En este contexto se plantea la hipótesis de alienación de intereses, la cual se basa en el argumento de que los intereses de los miembros de la familia y los accionistas externos a ésta se encuentran mejor 
alineados debido a los elevados niveles de propiedad en manos de la familia y su presencia a largo plazo en la compañía, lo cual minimiza los incentivos de la dirección a comportarse de forma oportunista. En este sentido, Wang (2006) argumenta que las compañías de propiedad familiar no tienen incentivos que se comporten de manera oportunista, pues la dirección adoptará políticas encaminadas a anular posibles daños a la reputación de la familia y mejorar su desempeño en el largo plazo. Bajo esta hipótesis, las empresas de propiedad familiar parecen tener un mejor desempeño y una mejor supervisión y control del equipo directivo, mitigando el oportunismo por parte de la dirección (Klein, 2002).

Si bien la empresa familiar presenta beneficios asociadas a su estructura de propiedad concentrada porque permite mitigar los costes de agencia generados por la separación entre propiedad y control, también presenta algunos inconvenientes derivados de esta estructura de propiedad. De acuerdo con Galve (2002), entre los principales se encuentran la oferta limitada de talento en la familia y problemas derivados del atrincheramiento. Con relación al mercado de talento directivo, la empresa se ve comprometida por la restricción del mantenimiento del control en manos del grupo familiar y por las restricciones de que las posiciones de dirección y control deben ser ocupadas por miembros de la familia (La Porta et al., 1999), lo que dificulta la contratación con base en la habilidad en la toma de decisiones. De hecho, Shliefer y Vishny (1997) sugieren que uno de los costes más grandes que los accionistas pueden imponer es permanecer activos en la dirección de la empresa aun cuando no son competentes o cualificados para dirigirla. Ante estas circunstancias, las familias afrontan el dilema de si abrirse o no al exterior incorporando a las personas que sí poseen los atributos demandados.

Por otro lado, como ya señalábamos, aunque la combinación de propiedad y control en una sola persona o familia puede reducir los costes de agencia, también esto podría tener su contraparte en el hecho de un excesivo protagonismo por parte del propietario en sus funciones directivas, lo que puede generar problemas de atrincheramiento. Es decir, cuando un accionista posee una cantidad importante de acciones de la empresa, los problemas de agencia se resuelven, pero no del todo debido a que el accionista puede usar su poder de voto para mejorar su situación particular en detrimento de otros accionistas. La hipótesis del atrincheramiento está basada en el argumento de que la concentración de propiedad crea incentivos para que los grandes accionistas o accionistas controladores expropien riqueza de los pequeños (Fama y Jensen, 1983; Shleifer y Vishny, 1997). En este sentido, un mayor conflicto de intereses se presentará ante un aumento en la participación de la dirección, basándose en que los gerentes que mantienen un porcentaje reducido de propiedad trabajarán de acuerdo con la maximización del beneficio debido a las fuerzas disciplinadoras que reducen la rentabilidad privada de la dirección; por el contrario, si los gerentes poseen un porcentaje importante, también poseen un poder de voto que garantizará su trabajo, pudiendo oponerse a un comportamiento maximizador de valor de la empresa imperando sus intereses como director (Delgado, 2003). 
La propiedad familiar como mecanismo de gobierno disciplinador de la

dirección en las empresas mexicanas: una evidencia empírica

Así, los propietarios-directivos pueden tomar ventajas de sus intereses personales a expensas de los propietarios externos. La propiedad privada, y en particular la familiar, incrementa el problema debido a que los derechos de propiedad y de autoridad formal se combinan con el status de familiar y con su resistencia a la incorporación de cambios en la empresa, lo que aumenta el riesgo de atrincheramiento de los directivos. Cuando la propiedad y, por tanto, el poder no están distribuidas simétricamente en la empresa, el objetivo del propietario principal de maximizar su función de utilidad puede conducirle a tomar acciones o decisiones que no vayan de acuerdo con el interés común y a adoptar un comportamiento oportunista y explotador con los accionistas minoritarios (Galve, 2002).

En lo que respecta a la concentración de la propiedad, ésta también constituye un importante mecanismo de control de la actuación directiva. Cuando se dispersa la propiedad, el control del accionista tiende a ser débil debido al problema parasitario supuesto, es decir, un accionista pequeño llevaría todos los costes de supervisión, pero comparte solamente una proporción pequeña de la ventaja, por lo que no estará interesado en ella. Si todos los accionistas pequeños se comportan de una manera similar, no habría una supervisión adecuada del equipo directivo, dándole suficiente margen para comportarse de manera oportunista; sin embargo, cuando se concentra la propiedad, los grandes accionistas podrían desempeñar un papel importante en la supervisión de la dirección (Abdul, 2004). Como señala Azofra y Santamaría (2002), en una organización en la que sea posible transferir los títulos de propiedad sin pérdidas de valor y en donde exista una importante dispersión del capital entre múltiples accionistas, éstos no cuentan con incentivos suficientes para controlar a la dirección; por el contrario, la existencia de un accionista mayoritario con capacidad para controlar y despedir a los directivos puede paliar posibles comportamientos oportunistas.

Finalmente, tenemos la participación accionarial del equipo directivo. La propiedad de acciones es una motivación importante para que los directivos tengan por objetivo maximizar el valor de la empresa para los accionistas, ya que los posibles comportamientos oportunistas por parte de éstos disminuirán cuanto más influyan sus acciones sobre su propia riqueza. Así, este mecanismo actúa de forma directa sobre las acciones realizadas por la dirección, con lo que la incorporación de propietarios en puestos de toma de decisiones tenderá a reducir políticas "sub-óptimas" de inversión (Muralanda, 2005). Desde esta perspectiva, podría esperarse un mayor valor de la empresa cuanto mayor sea la participación accionarial de este grupo; sin embargo, la participación de los directivos en la empresa presenta limitaciones como solución a los posibles comportamientos oportunistas. Por un lado, un directivo adverso al riesgo, con una gran proporción de su riqueza ligada al rendimiento de la empresa, puede decidir seguir políticas no maximizadoras de valor, pero que permitan limitar el riesgo; por otro, el mayor poder de voto de los directivos puede limitar la actuación supervisora del resto de los accionistas y del mercado al poseer éstos suficiente poder de voto para garantizar su continuidad (Fernández y Gómez-Ansón, 1999). De este modo, altas participaciones de los directivos pueden representar también comportamientos oportunistas. 


\subsubsection{Consejo de administración}

Como ya señalábamos, otro importante mecanismo de control que permite limitar la discrecionalidad por parte del equipo directivo es el consejo de administración. Podemos encontrar diferentes características que pueden influir en la efectividad con la que el consejo supervisa la actuación directiva en la empresa; de acuerdo con Fernández y GómezAnsón (1999) en la bibliografía se analizan básicamente dos características o variables que influyen en la capacidad supervisora del consejo: su composición y su tamaño.

En cuanto a la composición del consejo, se puede distinguir entre sus miembros dos grupos principales: los consejeros internos o propietarios - con un empleo o participación activa en la empresa - y los independientes o externos — no están vinculados a la dirección de la compañía. Se espera que estos últimos realicen una labor de supervisión del equipo directivo, ya que a los consejeros externos se les atribuye mayor independencia y, por lo tanto, mayor capacidad para controlar y evaluar a la dirección (Fama, 1980). De esta forma, se podría esperar una mayor eficiencia supervisora del consejo si cuenta con este tipo de consejeros, pues aunque los internos podrían aportar sus conocimientos y experiencia valiosa, éstos tenderán a ejercer una función supervisora de los directivos orientada hacia la maximización del valor de la empresa (García, 2003).

Como se puede apreciar en el párrafo anterior, la hipótesis de mayor efectividad del consejo de administración como órgano supervisor del equipo directivo deriva de una mayor presencia de consejeros independientes. En este contexto, cabría suponer que la concentración de propiedad en un pequeño grupo de personas se puede emplear como una forma de gobierno en la cual no fuese necesaria la presencia de consejeros independientes debido a que el accionista mayoritario ejerce la labor de supervisión del consejo. Sin embargo, este tipo de gobierno genera otros problemas como comentábamos, pues cuando la propiedad se encuentra altamente concentrada, en familias, por ejemplo, muchas veces miembros de la misma familia tienen una labor activa en la dirección de la empresa, lo que puede ocasionar que surjan conflictos de intereses entre el grupo controlador y los pequeños accionistas. Así, la incorporación de consejeros independientes, bajo estas circunstancias, asumirá las mismas funciones de supervisión hacia el grupo de control en interés de los accionistas minoritarios que cuando eran incorporados al consejo de administración para impedir los posibles comportamientos oportunistas por parte de la dirección (Salas, 2002).

Sin embargo, puede esperarse que este tipo de consejeros en empresas familiares no cumplan con su cometido debido a diferentes razones (Salas, 2002). Primero, si no tienen una participación en la empresa, el incremento del valor en ésta no tiene un gran impacto en su persona, además del coste de oportunidad asociado al tiempo que invierten en esta supervisión. Segundo, porque tal vez los consejeros externos deben su nombramiento al equipo directivo, dependiendo de este último su continuidad como parte del consejo de administración. De este modo, puede argumentarse que el nombramiento de consejeros independientes en las empresas que son controladas por familias estará influido por los 
La propiedad familiar como mecanismo de gobierno disciplinador de la

dirección en las empresas mexicanas: una evidencia empírica

posibles lazos personales del consejero independiente con la familia controladora y por la expectativa de que apoyarían a la dirección en las grandes decisiones; de esta forma, la independencia de los consejeros externos en las empresas controladas por familias podría verse empañada (Chen y Jaggi, 2000).

La segunda característica por considerar en relación con la eficiencia del consejo para ejercer su labor supervisora es su tamaño. La dimensión del consejo - medida por el número de consejeros - puede influir en la eficiencia supervisora por parte de éste. Esta influencia puede ser positiva o negativa. Positiva en el sentido de que un mayor número de consejeros facilitara esta tarea, ya que al haber más consejeros habría una mayor variedad de opiniones y podría conllevar un incremento de la capacidad de supervisión del consejo (Pearce y Zahra, 1992). Sin embargo, también podría suponer una influencia negativa, pues incrementos en el tamaño del consejo lo hacen menos efectivo debido a los problemas de coordinación e información que pudiesen surgir en el proceso de toma de decisiones, así como a la hora de disciplinar a la dirección (Jensen, 1993). La evidencia empírica, en este sentido, parece mostrar una relación negativa entre el tamaño del consejo y el valor de la empresa (Yermack, 1996; Fernández et al., 1998; Azofra et al., 2005).

\subsubsection{Endeudamiento}

La estructura financiera de la empresa y, en concreto, su nivel de apalancamiento pueden actuar igualmente como mecanismo supervisor de la dirección. Tradicionalmente, se han reconocido dos ventajas que acompañan a la decisión de incrementar el endeudamiento de la empresa: por un lado, la deuda al exigir unos pagos periódicos reduce los flujos de caja libres y genera incentivos para que la dirección tenga menores comportamientos de tipo discrecional (Jensen, 1986); por otro, un incremento de la deuda puede traducirse en una mejor decisión de inversión, puesto que la posibilidad de una quiebra en la empresa induce a los directivos a adoptar un comportamiento que trate de reducir la probabilidad de dicha quiebra. De esta manera, los directivos orientarán sus decisiones de forma más efectiva hacia el objetivo de creación de valor a fin de evitar la insolvencia y la quiebra empresarial, que perjudicará tanto a los accionistas como a los propios directivos (Fernández, 1999).

\section{Hipótesis y metodología}

\subsection{Hipótesis}

En cuanto a la estructura de propiedad, como ya se mencionó, los estudios relacionados con ésta han basado su análisis principalmente en tres aspectos: la concentración de propiedad, la identidad del propietario principal y la participación accionarial de los directivos como mecanismos de supervisión.

La concentración de la propiedad se percibe como un mecanismo natural de supervisión de la actuación directiva en la empresa. Los accionistas con una participación suficiente- 
mente significativa están dispuestos a asumir esta responsabilidad siempre que los beneficios esperados de la supervisión superen los costes derivados de su ejercicio (Díaz, 2000). Esta afirmación se fundamenta en la idea de que controlar la dirección les ocasiona a los accionistas atomísticos un gran esfuerzo de coordinación para obtener una pequeña parte de los beneficios derivados de tal supervisión. Si los accionistas poseen una cantidad importante de las acciones, tienen incentivos por desempeñar esta función debido a que pueden hacer cumplir sus derechos (García, 2003).

Otro aspecto por considerar en la limitación de la discrecionalidad por parte del equipo directivo es la identidad del propietario. En este sentido, Shleifer y Vishny (1986) argumentan que la existencia de grandes accionistas produce un mayor control de la dirección. Así, cuando el accionista principal es una familia, por ejemplo, los miembros de ésta actúan como supervisores de la actuación directiva logrando una mayor alineación de intereses entre accionistas y dirección, lo que se traduce en una mayor creación de valor en la empresa a través de los beneficios derivados de la mayor supervisión. Finalmente, como mecanismo supervisor encontramos la participación accionarial de los directivos. En este sentido, la empresa puede beneficiarse de la tenencia accionaria de los directivos como consecuencia de la alineación de intereses entre accionistas y dirección, lo cual contribuye a la reducción de la discrecionalidad por parte del equipo directivo. Sin embargo, los directivos que forman parte del accionariado de la empresa pueden beneficiarse de la posibilidad de atrincheramiento que les concede su mayor poder de control, ello les permite mantenerse en su puesto de trabajo y asegurarse su remuneración, lo que les daría un mayor margen para ejercer la discrecionalidad. La evidencia empírica, en este sentido, no es concluyente. Por tanto, la hipótesis que se plantea respecto a la estructura de propiedad es la siguiente:

H1: ceteris paribus, la discrecionalidad directiva será menor en empresas familiares que en empresas no familiares.

Con respecto al consejo de administración, como se ha expuesto previamente, la bibliografía sugiere que la presencia de miembros externos en el consejo establece un freno a las prácticas oportunistas por parte de la dirección. Estos consejeros representan los intereses de los accionistas: por un lado, los consejeros relacionados representan los intereses de los grupos accionariales importantes de la compañía, mientras que, por otro, los consejeros independientes representan los intereses de los accionistas minoritarios. El hecho de pertenecer al consejo de administración de la empresa da la oportunidad a estos grupos de ejercer un control directo sobre la actuación de equipo directivo (García y Gill, 2005).

Además de su composición, la eficiencia del consejo de administración en sus funciones de vigilancia y supervisión de los directivos se ha analizado por medio del tamaño del consejo. Los problemas de comunicación y coordinación inherentes a todo órgano de decisión colectivo se verán reducidos a medida que disminuye el número de sus integran- 
La propiedad familiar como mecanismo de gobierno disciplinador de la

dirección en las empresas mexicanas: una evidencia empírica

tes, por lo que se espera una mayor efectividad de las labores de supervisión del consejo cuanto menor sea su tamaño, lo que se verá plasmado en un menor uso del ejercicio de la discrecionalidad por parte de la dirección. De esta forma, las hipótesis que se plantean respecto a la composición y tamaño del consejo de administración son las siguientes:

H2: ceteris paribus, la discrecionalidad directiva está inversamente relacionada con la proporción de consejeros independientes y relacionados en el consejo de administración.

H3: ceteris paribus, la discrecionalidad directiva será mayor cuanto mayor sea el tamaño del consejo de administración.

Por otra parte, como comentábamos en el apartado 1.1.3, la estructura financiera de la empresa y, en concreto, su nivel de apalancamiento puede actuar como mecanismo supervisor de la actuación directiva. En principio, cuanto más endeudada esté la empresa más se reduce la discrecionalidad por parte de la dirección al disminuir los flujos de libre disposición (Jensen, 1986). Además, la mayor probabilidad de quiebra asociada a empresas con un alto grado de endeudamiento da lugar a un comportamiento más eficiente de los directivos con la finalidad de reducir dicha probabilidad y permanecer en su puesto de trabajo. Desde este punto de vista, formulamos una cuarta hipótesis:

H4: ceteris paribus, la discrecionalidad directiva será menor cuanto mayor sea el endeudamiento de la empresa.

El contraste de las hipótesis anteriores nos permite desarrollar nuestro primer objetivo: el análisis de la influencia de los mecanismos de gobierno - estructura de propiedad, consejo de administración y endeudamiento - sobre la discrecionalidad directiva tratando de identificar si la concentración de la propiedad, específicamente, el control familiar, resulta ser una variable relevante como mecanismos de supervisión y control del ejercicio de la discrecionalidad. Asimismo, debido a esta mayor supervisión que se les atribuye a las familias en estructuras de propiedad concentrada, los mecanismos de gobierno pueden presentar un funcionamiento diferente dependiendo de la estructura de propiedad. En este sentido, nos hemos planteado como segundo objetivo investigar si los mecanismos de gobierno se encuentran condicionados por la estructura de propiedad de la empresa.

Puede defenderse que el nombramiento de consejeros independientes en las empresas que son controladas por familias estará influido por los posibles lazos personales del consejero independiente con la familia controladora y por la expectativa de que apoyarían a la dirección en las grandes decisiones, por lo que la independencia de los consejeros externos en las empresas controladas por familias podría verse ensombrecida (Chen y Jaggi, 2000). En este sentido, el requerimiento de las prácticas de buen gobierno establecidas por la Bolsa Mexicana de Valores sobre la inclusión de consejeros independientes en los consejos de administración es motivado por el mejoramiento en el funcionamiento 
del equipo directivo de la compañía en lo referente a la transparencia desde el punto de vista del accionista, independientemente del control de la familia (Consejo Coordinador Empresarial, 1999). Por tanto, es de interés saber si la inclusión de consejeros externos en los consejos de administración está asociada con una menor discrecionalidad directiva cuando las empresas son poseídas y controladas por familias.

Por otro lado, la necesidad de acceso a la financiación externa por parte de la empresa y, en particular, la decisión de aumentar su deuda da lugar a una mayor supervisión y control de la misma por parte de los intermediarios financieros (Díaz, 2000). Además del control que se puede ejercer por parte de los propietarios, se le añade un control externo por parte de los tenedores de la deuda, los cuales no permitirán que ésta se destine a un uso con un fin indeterminado o excesivamente arriesgado (Suriñach et al., 2005). Sin embargo, la bibliografía parece mostrar que estructuras de propiedad concentrada, como lo son las empresas familiares, alivian los problemas de agencia evitando incurrir en mecanismos de control adicionales como la deuda. De este modo, planteamos una quinta hipótesis:

H5: El efecto de los mecanismos de gobierno sobre la discrecionalidad directiva dependerá de la estructura de propiedad de la empresa.

\subsection{Metodología}

\subsubsection{Los ajustes por devengo como medida de la discrecionalidad directiva}

Para contrastar las hipótesis planteadas en la sección anterior, es necesario definir una medida de la discrecionalidad directiva. De esta forma, y siguiendo la mayoría de la bibliografía sobre este tema, en este trabajo nos centramos en los ajustes por devengo como medida de la discrecionalidad directiva. ${ }^{1}$ En un trabajo que puede considerarse pionero en la línea de investigación sobre este tema, Jones (1991) propone un modelo de expectativas para separar los componentes discrecionales y no discrecionales de los ajustes por devengo totales. A pesar de que a lo largo de distintos trabajos especializados en la materia se han planteado diversos modelos alternativos, el modelo original de Jones sigue siendo el más utilizado en esta línea de investigación. Éste es también el modelo utilizado en el análisis desarrollado en este trabajo.

El modelo propuesto por Jones, que se presenta en la expresión (1), regresa los ajustes por devengo observados (ADT) sobre dos variables: cambio en las ventas $(\triangle \mathrm{REV})$, que controla el componente normal de los ajustes por devengo de circulante; y el nivel de inmovilizado bruto (GPPE), que modela el componente no discrecional del gasto por depreciación y amortización del periodo. El signo esperado del coeficiente de GPPE es negativo, ya que

\footnotetext{
1 De acuerdo a García y Gill (2005), la bibliografía distingue básicamente dos mecanismos de naturaleza contable para alterar el resultado declarado: los cambios en los procedimientos de reconocimiento y valoración de los elementos patrimoniales, y los ajustes por devengo, derivados de las decisiones de imputación de determinados gastos e ingresos al resultado a lo largo del tiempo. El primer mecanismo resulta más transparente para el usuario, ya que existe obligación de justificar los cambios contables en la memoria; por ello, suele considerarse que la gerencia estimará más atractiva la manipulación de los ajustes por devengo.
} 
La propiedad familiar como mecanismo de gobierno disciplinador de la

dirección en las empresas mexicanas: una evidencia empírica

estará relacionado con el gasto por depreciación. Sin embargo, el signo esperado del coeficiente de la variación en la cifra de negocios no resulta tan evidente dado que esta variación puede conllevar cambios que supongan aumento o disminución en distintas partes del circulante (Delgado, 2003). Ambas variables, así como la constante, se dividen entre el activo total para evitar problemas de heterocedasticidad. ${ }^{2}$ De esta forma, los ajustes por devengo no discrecionales (ADND) son las predicciones de la estimación por mínimos cuadrados ordinarios (MCO) de la regresión (1), mientras que los ajustes por devengo discrecionales (ADD) son los residuos de la misma.

$$
A D T=\frac{\beta_{0}}{A_{i t-1}}+\beta_{1}\left(\begin{array}{c}
\Delta R E V_{i t} \\
A_{i t-1}
\end{array}\right)+\beta_{2}\left(\begin{array}{c}
G P P E_{i t} \\
A_{i t-1}
\end{array}\right)+\varepsilon_{i t}
$$

Los ajustes por devengo totales observados (ADT) están definidos por el cambio en activo circulante $(\triangle \mathrm{AC})$, excepto el cambio en tesorería e inversiones financieras temporales $(\triangle \mathrm{TES})$, menos el cambio en el pasivo circulante $(\triangle \mathrm{PC})$, exceptuando el cambio en la deuda financiera $(\triangle \mathrm{DEBT})$, y menos el gasto en amortización y depreciación a largo plazo del periodo (AMORT); A representa el activo total, $e$ es el término de error del modelo, $i$ y $t$ son los subíndices representativos de la empresa y el año respectivamente.

Siguiendo a Delgado (2003), en la estimación del modelo de Jones de corte transversal hemos incluido n-1 variables dummys representativas de $\mathrm{n}$ sectores para así poder efectuar la estimación utilizando todas las observaciones, ya que el número de empresas por sector es muy reducido (media 17.5). Para llevar a cabo la estimación en el modelo de Jones presentado previamente, es necesaria la información de las variables contables que intervienen en el mismo para el mayor número de empresas posibles para el año en cuestión y sector de actividad. De acuerdo con García y Gill (2005), para llevar a cabo la estimación es necesario disponer de al menos seis observaciones para cada sector. ${ }^{3}$

\subsubsection{Modelo de regresión y definición de variables}

Una regresión por medio de mínimos cuadrados ordinarios (MCO) se utiliza para probar la asociación entre la variable dependiente y las explicativas, donde la medida de la discrecionalidad directiva, el valor absoluto de los ajustes por devengo discrecionales $(|\mathrm{ADD}|)$, es la variable dependiente y como variables independientes se incluyen las relacionadas con la estructura de gobierno de la empresa. El modelo de regresión múltiple es:

$$
\begin{aligned}
& \mid \operatorname{ADD}_{\mid \mathrm{i}}=\beta_{0}+\beta_{1} \mathrm{~F}_{-} \text {FAM }_{\mathrm{i}}+\beta_{2} \text { CFAM }_{\mathrm{i}}+\beta_{3} \text { ENDEU }_{\mathrm{i}}+\beta_{4} \text { LNCONS }_{\mathrm{i}}+\beta_{5} \text { CONIND }_{\mathrm{i}}+\beta_{6} \\
& \mathrm{CONREL}_{\mathrm{i}}+\beta_{7} \mathrm{EDAD}_{\mathrm{i}}+\beta_{8} \text { LTAM }_{\mathrm{i}}+\varepsilon_{\mathrm{i}}
\end{aligned}
$$

2 Muchos trabajos han utilizado el modelo de Jones original sin deflactar la constante argumentando que no hay razón teórica para hacerlo; sin embargo, los resultados de este trabajo no varían sensiblemente al utilizar este planteamiento alternativo.

3 Según García y Gill (2005), en su trabajo sobre las prácticas de earnings management, hacen una amplia revisión de la bibliografía sobre este tema estableciendo que al menos seis observaciones por sector y año es lo habitual. 


\section{Donde:}

\begin{tabular}{|c|c|}
\hline$|\mathrm{ADD}|$ & Valor absoluto de los ajustes por devengo discrecional del modelo de Jones. \\
\hline F_FAM & $\begin{array}{l}\text { Variable dummy que toma valor de } 1 \text { si una familia controla más del } 50 \% \text { de las acciones } \\
\text { de la empresa y } 0 \text { si no lo está. }\end{array}$ \\
\hline CFAM & $\begin{array}{l}\text { Variable dummy que toma el valor de } 1 \text { si la dirección de la empresa se encuentra en manos } \\
\text { de un miembro de la familia controladora y } 0 \text { si no lo está. }\end{array}$ \\
\hline F_FAMCFAM & Variable creada a partir de la interacción entre F_FAM y CFAM. \\
\hline ENDEU & Endeudamiento de la empresa, medido por el pasivo total entre el activo total. \\
\hline LNCONS & Tamaño del consejo, determinado por el logaritmo del número de consejeros. \\
\hline CONIND & Número de consejeros independientes entre el número total de consejeros. \\
\hline CONREL & Número de consejeros relacionados entre el número total de consejeros. \\
\hline EDAD & Número de años de la empresa. \\
\hline LTAM & Logaritmo del activo total. \\
\hline
\end{tabular}

Las variables de gobierno corporativo son estructura de propiedad (F_FAM), dirección de la empresa (CFAM), la composición del consejo de administración: consejeros independientes (CONIND) y consejeros relacionados (CONREL), el tamaño del consejo (NCONS) y el endeudamiento (ENDEU). Con respecto a las variables de control, y con base en lo realizado en trabajos previos (Warfield et al., 1995; Delgado, 2003; Wang, 2006), hemos incluido el tamaño de la empresa (TAM) y el número de años de la empresa (EDAD), el cual es controlada, pues las empresas más viejas son menos probables a ser de carácter familiar (Wang, 2006).

\subsubsection{Muestra}

En la muestra utilizada en este trabajo de investigación se toma como punto de partida aquellas empresas que cotizaron en la Bolsa Mexicana de Valores (BMV) durante los años 2003 y 2004. Diferenciamos entre dos muestras: la primera de ellas es la utilizada en la estimación de los ajustes por devengo y la segunda es aquella usada en la construcción del modelo explicativo de la discrecionalidad directiva propuesto en este trabajo. La fuente de información utilizada para la obtención de los estados financieros de las empresas es la base de datos "Infosel". La información relativa a la estructura de propiedad y gobierno de las compañías de la muestra utilizada en el análisis fue obtenida de los reportes anuales de las empresas publicados por la BMV en su página $w^{4} b^{4}$ correspondientes a los ejercicios del 2003 y 2004. Las entidades financieras fueron excluidas por las peculiaridades que presenta el proceso de generación de sus ajustes por devengo, lo que requerirá un análisis particularizado de las mismas. En principio, se disponía de 122 empresas, de las cuales se eliminaron 17 al no contener información suficiente en sus estados financieros debido a que han desaparecido o ya no cotizan en bolsa, por lo cual no hay información disponible para los años objeto de estudio. Las 105 empresas que componen la muestra final pueden considerarse representativas del tejido empresarial mexicano debido a los distintos sectores que en dicha muestra se encuentran.

\footnotetext{
4 Véase http://www.bmv.com.mx
} 
La propiedad familiar como mecanismo de gobierno disciplinador de la

dirección en las empresas mexicanas: una evidencia empírica

\section{Resultados}

\subsection{Estadística descriptiva}

\section{a) Variable dependiente}

En la tabla 1, aparece la estadística descriptiva de los ADT y ADD estimados para la muestra objeto de análisis. Al igual que en otros trabajos (Warfield et al., 1995; Delgado, 2003; García y Gill, 2005; Wang, 2006), la media de los ajustes por devengo totales adopta un valor negativo ( $2 \%)$, mientras que — como podía esperarse - la media de los ajustes por devengo discrecionales para la muestra utilizada en la estimación es cero. Este resultado sugiere que no existe manipulación sistemática a la alza ni a la baja en las observaciones de la muestra, por lo que al igual que en otros trabajos en esta línea de investigación, se considera el valor absoluto de los ajustes por devengo discrecionales $(|\mathrm{ADD}|)$ como medida de la discrecionalidad directiva (García y Gill, 2005).

Tabla 1

Estadística descriptiva de los ajustes por devengo discrecionales estimados con el modelo de Jones

\begin{tabular}{c|c|c|c|c}
\hline \hline Variable & Media & Desv. Típica & Mínimo & Máximo \\
\hline \hline Ajustes totales & -0.02 & 0.10 & -0.27 & 0.58 \\
\hline Ajustes discrecionales & 0.00 & 0.08 & -0.20 & 0.38 \\
\hline \hline
\end{tabular}

\section{b) Variables explicativas}

La estadística descriptiva de las variables usadas para la estimación empírica, exceptuando la de propiedad familiar, se presenta en la tabla 2, dividida por empresas familiares y no familiares. El promedio de accruals anormales (ADD) es de 0.061 para empresas familiares y de 0.066 para las empresas no familiares, lo cual nos indica, a primera vista, que la discrecionalidad tiende a ser mayor en aquellas empresas que no son de carácter familiar. La variable de endeudamiento (ENDEU) muestra un valor de 0.51 para empresas familiares, mientras que para las empresas no familiares presenta un valor de 0.50 , lo que nos muestra que, en promedio, las empresas familiares están un poco más endeudadas que las no familiares. La variable porcentaje de consejeros independientes (CONIND) muestra que, en promedio, ambas poseen la misma cantidad de consejeros independientes en sus consejos. Las empresas familiares presentan en promedio un menor porcentaje de consejeros relacionados (CONREL). La variable número de consejeros (NCONS) nos indica que los consejos de administración de las empresas no familiares son en promedio un poco más grandes que los consejos de las empresas familiares. Por último, las variables de control logaritmo del activo total de la empresa (LTAM) es de 15.80 para empresas familiares y de 15.41 para las no familiares, y la edad (AGE) es de 29.83 para em- 
presas familiares y de 32.02 para no familiares, es decir, las empresas no familiares son más viejas que las familiares.

Tabla 2

Estadística descriptiva de las variables del análisis de regresión

Empresas familiares

\begin{tabular}{ccccc}
\hline \hline Variable & Media & $\begin{array}{c}\text { Desv. } \\
\text { típ. }\end{array}$ & Mín. & Máx. \\
\hline \hline ADD & 0.06 & 0.05 & 0 & 0.20 \\
ENDEU & 0.51 & 0.25 & 0.04 & 1.51 \\
CONIND & 0.33 & 0.22 & 0 & 0.75 \\
CONREL & 0.09 & 0.17 & 0 & 0.8 \\
NCONS & 10.33 & 3.71 & 3 & 20 \\
EDAD & 29.83 & 20.73 & 5 & 105 \\
LTAM & 15.81 & 1.72 & 11.91 & 19.39 \\
\hline \hline
\end{tabular}

Empresas no familiares

\begin{tabular}{ccccc}
\hline \hline Variable & Media & $\begin{array}{c}\text { Desv. } \\
\text { típ. }\end{array}$ & Mín. & Máx. \\
\hline \hline ADD $\mid$ & 0.06 & 0.05 & 0.003 & 0.20 \\
ENDEU & 0.50 & 0.18 & 0.05 & 0.9 \\
CONIND & 0.33 & 0.25 & 0 & 0.92 \\
CONREL & 0.14 & 0.19 & 0 & 0.83 \\
NCONS & 10.81 & 3.89 & 5 & 19 \\
EDAD & 32.02 & 21.54 & 5 & 81 \\
LTAM & 15.41 & 1.95 & 11.13 & 19.08 \\
\hline \hline
\end{tabular}

\subsection{Resultados del análisis de regresión}

\section{a) Estimación de los ajustes por devengo}

La tabla 3 muestra los resultados de la estimación del modelo de Jones de corte transversal. En la tabla, aparece el coeficiente correspondiente, así como el estadístico t y el coeficiente del $\mathrm{R}^{2}$. Puede observarse cómo los coeficientes presentan el signo esperado, positivo para $\triangle \mathrm{REV}$ y negativo para GPPE, además de ser significativos.

Tabla 3

Estimación del modelo de Jones

$\mathrm{ADT}=\beta_{0}+\beta_{1} \mathrm{GPPE}+\beta_{2} \triangle \mathrm{REV}$

\begin{tabular}{cl}
\hline \hline & ADT \\
\hline \hline AREV & 0.174 \\
& $(3.18)^{* *}$ \\
GPPE & -0.051 \\
& $(2.54)^{*}$ \\
\hline \hline $\mathrm{R}^{2}$ & 0.29 \\
\hline \hline
\end{tabular}

Valor absoluto del t estadístico en paréntesis

+ Significativo al $10 \%, *$ Significativo al $5 \%$,

** Significativo al $1 \%$

Habiendo estimado el modelo de Jones, obtenemos la variable dependiente del modelo, con lo cual pasaremos al contraste de las hipótesis planteadas en la sección anterior, sirviéndonos del análisis de regresión mediante la estimación por mínimos cuadrados ordinarios. 
La propiedad familiar como mecanismo de gobierno disciplinador de la dirección en las empresas mexicanas: una evidencia empírica

\section{b) Resultados de la estimación}

Los resultados del análisis de regresión simple, utilizando como medida de la discrecionalidad el valor absoluto de los ajustes por devengo obtenidos mediante el modelo de Jones, aparecen en la tabla 4 . Por otra parte, la tabla 5 muestra los resultados correspondientes al análisis de regresión con la muestra dividida en empresas familiares y no familiares.

Tabla 4

Resultados de la estimación del modelo global

\begin{tabular}{cc}
\hline \hline & $\mid$ ADD \\
\hline \hline F_FAMCFAM & -0.027 \\
ENDEU & $(2.64)^{* *}$ \\
& 0.040 \\
CONIND & $(2.00)^{*}$ \\
& -0.051 \\
CONREL & $(2.53)^{* *}$ \\
& -0.060 \\
LNCONS & $(2.22)^{*}$ \\
& -0.022 \\
EDAD & $(1.59)$ \\
& -0.000 \\
LTAM & $(1.10)$ \\
& -0.001 \\
CONSTANTE & $(0.50)$ \\
& 0.156 \\
R & $(3.77)^{* *}$ \\
\hline \hline & 0.23 \\
\hline \hline Valor absoluto del t estadístico en paréntesis \\
+Significativo al 10\%, *Significativo al 5\%, \\
* Significativo al 1\%
\end{tabular}

Tabla 5

Resultados de la estimación del modelo individual

Empresas familiares

\begin{tabular}{|c|c|}
\hline & $|\mathbf{A D D}|$ \\
\hline ENDEU & $\begin{array}{c}0.050 \\
(2.08)^{*}\end{array}$ \\
\hline CONIND & $\begin{array}{l}-0.069 \\
(2.34)^{*}\end{array}$ \\
\hline CONREL & $\begin{array}{c}-0.074 \\
(1.96)^{+}\end{array}$ \\
\hline LNCONS & $\begin{array}{l}-0.026 \\
(1.35)\end{array}$ \\
\hline EDAD & $\begin{array}{l}-0.000 \\
(0.89)\end{array}$ \\
\hline LTAM & $\begin{array}{l}0.002 \\
(0.61)\end{array}$ \\
\hline CONSTANTE & $\begin{array}{l}0.094 \\
(1.53) \\
\end{array}$ \\
\hline $\mathrm{R}^{2}$ & 0.28 \\
\hline $\begin{array}{l}\text { or absoluto del } \mathrm{t} \\
\text { éntesis } \\
\text { gnificativo al } 10 \% \\
\%, * * \text { Significati }\end{array}$ & $\begin{array}{l}\text { ico en } \\
\text { ificativo } \\
0\end{array}$ \\
\hline
\end{tabular}

Empresas no familiares

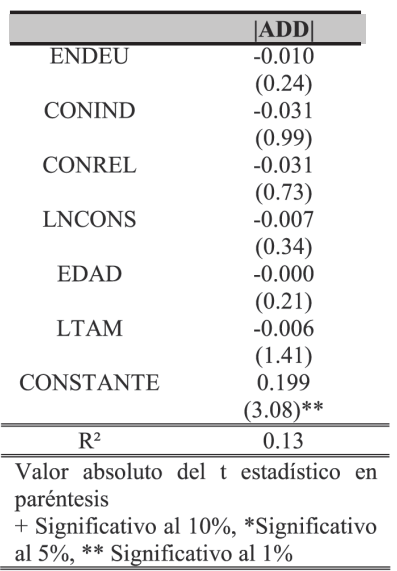


Como podemos apreciar en la tabla 4, consistente con la evidencia presentada, los resultados obtenidos confirman la primera hipótesis planteada $(H 1)$ : hay una relación negativa entre la discrecionalidad directiva y la variable de interacción entre estructura de propiedad familiar y dirección de la empresa en manos de un miembro de la familia (F_FAMCFAM), que al ser estadísticamente significativa es consistente con la idea de un menor ejercicio de la discrecionalidad directiva a medida que la propiedad de la empresa se encuentra en manos de una familia, a la vez que algún miembro de ésta la dirige. ${ }^{5}$ Así, la evidencia obtenida parece mostrar que en países como México, donde existe una baja protección de los inversionistas, la mejor forma de protegerse parece ser eliminando el problema de tipo free-rider, aumentando la concentración en la propiedad de la empresa y generando algún tipo de accionista controlador, ya que de acuerdo con la investigación en gobierno corporativo internacional se argumenta que este diferente grado de concentración de la propiedad es una respuesta racional y valiosa ante un sistema que no protege los intereses de los inversores minoritarios.

La segunda hipótesis (H2) se establecía con el objeto de conocer cómo afecta la composición del consejo de administración a la utilización de ajustes por devengo discrecionales. Efectivamente, los resultados confirman la segunda hipótesis planteada al mostrar una relación negativa entre el porcentaje de consejeros independientes y relacionados en el consejo de administración y la utilización de ajustes por devengo discrecionales, además de ser ésta estadísticamente significativa. Tenemos, pues, una fuerte evidencia de que entre mayor sea el número de consejeros independientes y relacionados en el consejo de administración, una menor discrecionalidad directiva habrá y, por ende, un mejor desempeño de las labores de supervisión encomendadas al consejo. Los resultados obtenidos en el modelo individual para empresas familiares presentan también una relación negativa y significativa de estas variables con la dependiente. En cuanto al modelo individual para empresas no familiares, a pesar de no resultar significativas, los signos de estas variables siguen siendo negativos, lo cual muestra la importancia de este tipo de consejeros como medida de supervisión y control de la actuación directiva tanto en empresas familiares como no familiares.

En cuanto al número de consejeros, la variable no resulta significativa, además de que los resultados obtenidos son opuestos a la hipótesis planteada (H3), pues el ejercicio de la discrecionalidad directiva disminuye a medida que aumenta el tamaño del consejo de administración, medido por el logaritmo del número de consejeros. Cabe señalar que se llevó a cabo la estimación con la variable número de consejeros (NCON) sin transformación logarítmica con el fin de ver si los resultados se modificaban; aunque las estimaciones no se muestran, no se presentaron cambios en los resultados de ésta.

Para la evidencia de la relación entre endeudamiento y discrecionalidad directiva, de acuerdo con la hipótesis planteada $(H 4)$, es de suponer una relación negativa entre deuda y dis-

\footnotetext{
${ }^{5}$ Cabe mencionar que se realizó la regresión con las variables F_FAM y CFAM por separado y aunque presentaron el signo esperado, negativo para F_FAM y CFAM, no resultaron ser significativas.
} 
La propiedad familiar como mecanismo de gobierno disciplinador de la

dirección en las empresas mexicanas: una evidencia empírica

crecionalidad directiva. Sin embargo, la variable presenta signo positivo, lo que muestra mayores incentivos por parte de la dirección por utilizar los ajustes por devengo discrecionales a medida que el ratio de endeudamiento de la empresa aumenta. Este mismo signo se presenta cuando estimamos el modelo individual considerando sólo empresas familiares. No obstante, cuando sólo consideramos empresas que no son de carácter familiar el signo, a pesar de no ser significativo, se vuelve negativo, lo que indica una disminución del ejercicio de la discrecionalidad en este tipo de empresas a medida que ésta se encuentra más endeudada. La explicación que nosotros consideramos para esto es la siguiente: los problemas de agencia y las asimetrías de información difieren en función del entorno institucional en el que la empresa desarrolla su actividad. La estructura de propiedad juega un papel importante en los conflictos de agencia, tanto entre accionistas y directivos como entre accionistas y acreedores. En el caso de las empresas mexicanas, observamos una importante concentración de la propiedad que, según señala Jensen (1986), facilita una mejor supervisión de las actividades de la empresa por parte de los principales accionistas (familias), con lo que reducen así el conflicto de intereses entre principal y agente. Por lo tanto, debido a esta supervisión por parte de los miembros de la familia, cabría esperar que los propietarios de las empresas familiares, al ejercer una fuerte supervisión sobre el equipo directivo, tendrían incentivos a expropiar riqueza de los acreedores, ya que éstos ya ejercen las labores de supervisión y no necesitan de la ayuda de alguien más para desempeñar esta labor, con lo cual la dirección tenderá hacia la expropiación de riqueza, por parte de los proveedores de fondos financieros, en beneficio de los accionistas. Es decir, la capacidad de supervisión de los accionistas mayoritarios, derivado de la concentración de propiedad, influirá en los directivos incentivándolos hacia la adopción de inversiones más arriesgadas para beneficiar a los accionistas a costa de los prestamistas. Por otro lado, tenemos la estructura de propiedad menos concentrada, en la cual los accionistas, al no poseer una cantidad importante de acciones de la empresa, no tienen incentivos para desempeñar las labores de supervisión de la actuación directiva, por lo que en estas empresas, debido a su estructura de propiedad, será necesario establecer mecanismos que ayuden en la supervisión y control de la dirección. En este sentido, la estructura financiera de la empresa y, en concreto, su nivel de apalancamiento, pueden actuar como mecanismo supervisor de la dirección, ya que conforme la deuda aumenta la supervisión sobre el equipo directivo se incrementa de igual forma debido a que la institución otorgante de fondos se convierte en un supervisor más de la actuación directiva. De esta forma, distinguimos entre dos tipos de mecanismos internos que la empresa puede utilizar para alinear los intereses de la dirección con los accionistas: la concentración de propiedad y el endeudamiento; éstos ejercen una influencia distinta sobre la discrecionalidad directiva dependiendo de la estructura de propiedad de la empresa. Como señala Coles et al. (2001), se produce un efecto de sustitución entre ambos mecanismos de tal manera que la empresa que no utilice la concentración de la propiedad, en familias, por ejemplo, deberá hacer más énfasis en mecanismos como el endeudamiento si quiere obtener un mejor resultado y, viceversa, aquellas empresas en las que la propiedad se encuentre concentrada deberán recurrir en menor medida al endeudamiento como mecanismo de supervisión sobre la dirección. 
Finalmente, con respecto a las variables de control incluidas en el modelo, tamaño y edad, tienen en todo caso un coeficiente negativo y no resultan ser significativas en nuestros resultados.

\section{Conclusiones}

Este trabajo presenta evidencia empírica sobre la relación entre los mecanismos de gobierno de la empresa y la discrecionalidad directiva, así como si estos mecanismos se encuentran condicionados por la estructura de propiedad. Nuestro análisis se ha centrado en tres mecanismos de gobierno fundamentalmente: la estructura de propiedad, considerada como familiar o no familiar; la composición y tamaño del consejo de administración; y el nivel de endeudamiento de la empresa. Para ello, nos hemos basado en una muestra de 105 empresas mexicanas que cotizaron en bolsa durante los periodos 2003 y 2004.

Los resultados obtenidos en el modelo global corroboran la evidencia presentada previamente y sugieren una menor discrecionalidad directiva en empresas familiares que en empresas no familiares. También se observa que la presencia de consejeros independientes - representantes de los accionistas minoritarios - y relacionados - representantes de grupos accionariales importantes - en el consejo de administración tiende a disminuir el ejercicio de la discrecionalidad. Adicionalmente, encontramos que la relación entre el nivel de endeudamiento y el ejercicio de la discrecionalidad directiva es positiva para estructuras de propiedad familiar y negativa para estructuras de propiedad difusa, lo que nos indica que en estructuras de propiedad concentradas, como la familiar, los grandes propietarios al realizar las labores de supervisión de la dirección reducen el conflicto de intereses entre accionistas y directivos, con lo que estos accionistas mayoritarios pueden imponer sus intereses en la toma de decisiones a los directivos, incitándolos a efectuar una transferencia de riqueza en detrimento de los acreedores, mientras que en estructuras de propiedad atomizada los propietarios al no poseer una cantidad importante de acciones de la empresa no tienen incentivos para realizar labores de supervisión sobre la dirección, con lo cual es necesario establecer mecanismos que ayuden a desempeñar esta función. En este caso, la estructura financiera de la empresa, específicamente su nivel de endeudamiento, actúa como mecanismo supervisor del equipo directivo. Hablamos, pues, de mecanismos que se sustituyen dependiendo de la estructura de propiedad de la empresa porque si bien en los análisis iniciales la deuda se contempla como un mecanismo de supervisión de la discrecionalidad directiva, la evidencia empírica parece mostrar que la deuda se presenta como un mecanismo de supervisión solo cuando la concentración de la propiedad es baja.

En cuanto a las limitaciones del estudio, en el presente trabajo se han de valorar con prudencia los resultados expuestos, pues el tipo de metodología empleada podría no considerarse la más adecuada debido al carácter estático del estudio, determinado por la ausencia de un análisis de cómo los mecanismos de gobierno pueden afectar la discrecionalidad directiva en las empresas a lo largo del tiempo, es decir, modelos con base de datos más amplios 
La propiedad familiar como mecanismo de gobierno disciplinador de la

dirección en las empresas mexicanas: una evidencia empírica

(datos panel o series de tiempo) podrían incorporar efectos temporales en la estimación, lo que nos proporcionaría una visión más amplia de los resultados.

Asimismo, sería importante tener en cuenta el análisis de la interacción entre los mecanismos de gobierno, estudiado mediante el uso de técnicas estadísticas como ecuaciones estructurales, con el fin de poder analizar la importancia de éstos de forma conjunta y ver si difiere sustancialmente del análisis individual. En este sentido, para dar continuidad a este trabajo y como futuras líneas de investigación, nos parece muy interesante profundizar en el análisis conjunto de los distintos mecanismos de gobierno, lo cual puede abrir nuevos caminos en los que la investigación sobre gobierno de la empresa incorpore esa consideración de interdependencia entre los distintos mecanismos de gobierno.

\section{BIBLIOGRAFÍA}

ABDUL, F., (2004): "Corporate Governance and Ownership Structure in the Malaysian Corporate Sector", en Advances in Financial Economics, no. 9, septiembre, pp. 355-385.

AGRAWAL, A. y C. R. KNOEBER, (1996): "Firm Performance and Mechanisms to Control Agency Problems between Managers and Shareholders", en Journal of Financial and Quantitative Analysis, vol. 31, no. 3, septiembre, pp. 337-398.

AZOFRA, V., ANDRÉS de, P. y F. LÓPEZ, (2005): “Corporate Boards in OECD Countries: Size, Composition, Functioning and Effectiveness", en Corporate Governance: An International Review, vol. 13, no. 2, marzo, pp.197-210.

AZOFRA, V. y M. SANTAMARÍA, (2002): “Gobierno y eficiencia de las cajas de ahorros españolas”, en Universia, Business Review, no. 002, segundo trimestre, pp. 48-59.

BERLE, A. A. y G. D. MEANS, (1932): “The Modern Corporation and Private Property”, Macmillan, New York.

BUSHMAN, R. y A. SMITH, (2001): "Financial Accounting Information and Corporate Governance", en Journal of Accounting and Economics, vol. 32, no. 3, diciembre, pp. 237-333.

CABRERA-SUAREZ, K., P. DE SAÁ-PÉREZ, y D. GARCÍA-ALMEIDA, (2000): “The Sucesión Process from a Resource — and Knowledge - Based View of the Family Firm”, en Family Business Review, Vol. XIV, No. 1, pp. 37-47.

CASSON, M., (1999): “The Economics of the Family Firm”, en Scandinavian Economic History Review, vol. 47, no. 1, febrero, pp.10-23.

CHAMI, R., (1999): "What's Different about Family Business?", Working paper, Trabajo publicado en la serie del Fondo Monetario Internacional "Artículos de Trabajo" con el número 01/70. 
CHEN, J. P. y B. JAGGI, (2000): “Association between Independent Nonexecutive Directors, Family Control and Financial Disclosures in Hong Kong”, en Journal of Accounting and Public Policy, vol. 19, no.4, junio, pp. 285-310.

COLES, J.W., V. B. McWILLIAMS y N. SEN, (2001): “An Examination of the Relationship of Governance Mechanisms to Performance”, en Journal of Management, vol. 27, no.1, abril, pp.23-50.

CONSEJO COORDINADOR EMPRESARIAL, (1999): Código de mejores prácticas corporativas, Consejo Coordinador Empresarial, México, D.F.

CUESTA, J. V., (2000): "Mecanismos jurídicos para garantizar la continuidad de la empresa familiar", en el I Congreso Nacional de Investigación sobre Empresa Familiar, ponencias y comunicaciones, Organismo Público Valenciano de Investigación, pp. 229-242.

DELGADO, M. M., (2003): Factores determinantes de la discrecionalidad contable: Una aplicación empírica a las empresas cotizadas españolas, Servicio de Publicaciones Universidad de Burgos, Burgos, España.

DÍAZ, B., (2000): Comportamiento supervisor y beneficios privados de la propiedad accionarial: Un análisis empírico para el caso español, tesis doctoral, Universidad de Cantabria.

FAMA, E. F., (1980): “Agency Problems and the Theory of the Firm”, en Journal of Political Economy, no. 88, abril, pp. 288-307.

y M. C. JENSEN, (1983): "Separation of Ownership and Control”, en Journal of Law and Economics, no. 26, junio, pp. 301-325.

FERNÁNDEZ, A., S. GÓMEZ-ANSÓN, y C. FERNÁNDEZ, (1998): “El papel supervisor del consejo de administración sobre la actuación gerencial. Evidencia para el caso español", en Investigaciones Económicas, no. 22, septiembre, pp. 501-516.

FERNÁNDEZ, A. I., y S. GÓMEZ, (1999): “Un estudio de las ofertas públicas de adquisición en el mercado de capitales español”, en Investigaciones Económicas, vol. 23, no. 3, septiembre, pp. 471-495.

FERNANDEZ, P., (1999): Valoración de empresas, Gestión 2000, Barcelona.

GALVE, C., (2002): “Propiedad y gobierno: la empresa familiar”, en Ekonomiaz, Universidad de Zaragoza, no. 50, segundo cuatrimestre, pp. 129-152.

GARCÍA, M. G., (2003): El gobierno corporativo y las decisiones de crecimiento empresarial: evidencia en las cajas de ahorro españolas, tesis doctoral, Universidad de las Palmas de Gran Canaria. 
La propiedad familiar como mecanismo de gobierno disciplinador de la

dirección en las empresas mexicanas: una evidencia empírica

GARCÍA, O. y GILL, B. (2005): "El gobierno corporativo y las prácticas de earnings management: evidencia empírica en España”, Working Papers. Serie EC, Instituto Valenciano de Investigaciones Económicas (IVIE), abril, WP-EC 2005-11.

GEDAJLOVIC, E. y D. SHAPIRO, (1998): "Management and Ownership Effects: Evidence from Five Countries", en Strategic Management Journal, vol. 19, no. 6, junio, pp. 533-553.

GISPERT, C. y P. ORTÍN, (2002): "Mecanismos de disciplina de los directivos en España: El consejo de administración y los sistemas de retribución", en Ekonomiaz, Universidad de Zaragoza, no. 50, segundo cuatrimestre, pp. 138-157.

JENSEN, M. C. y W. H. MECKLING, (1976): "Theory of the Firm: Managerial Behaviour, Agency Costs and Ownership Structure", en Journal of Financial Economics, vol. 3, no.4, octubre, pp. 305-360.

JENSEN, M. C., (1986): "Agency Costs and Free Cash Flow, Corporate Finance, and Takeovers", en The American Economic Review, no. 76, mayo, pp. 323-329.

(1993): "The Modern Industrial Revolution, Exit, and the Failure of Internal Control Systems", en Journal of Finance, vol. 48, no. 3, julio, pp. 831-880.

JONES, J., (1991): "Earnings Management During Import Relief Investigations", en Journal of Accounting Research, vol. 29, no. 2, otoño, pp. 193-233.

KINI, O., W. KRACAW y S. MIAN, (1995): "Corporate Takeovers, Firm Performance, and Board Composition", en Journal of Corporate Finance. vol. 1, no. 3, abril, pp. 383-412.

KLEIN, A., (2002): "Audit Committee, Board of Director Characteristics, and Earnings Management", en Journal of Accounting and Economics, vol. 33, no. 3, agosto, pp. $375-400$.

LA PORTA, R. et al., (1998): “Law and Finance”, en Journal of Political Economy, vol. 106, no. 6, diciembre, pp.1113-1155.

, (2000): "Investor Protection and Corporate Governance", en Journal of Financial Economics, vol. 58, no.2, octubre, pp. 3-27.

LA PORTA, R., F. LÓPEZ-DE-SILANES y A. SHLEIFER, (1999): “Corporate Governance around the World", en Journal of Finance, vol. 14, no. 2, abril, pp. 471-517.

PEARCE, J. A. y S. A. ZAHRA, (1992): "Board Compensation from a Strategic Contingency Perspective", en Journal of Management Studies, no. 29, abril, pp. 411-438.

SALAS, V., (2002): "El gobierno de la empresa", en Colección de Estudios Económicos de la Caixa, no. 29, septiembre, Barcelona: Servicio de estudios de La Caixa, pp. 5-214. 
SHLEIFER, A. y R. W. VISHNY, (1986): "Large Shareholders and Corporate Control", en Journal of Political Economy, vol. 94, no. 3, junio, pp. 461-488.

, (1997): “A Survey of Corporate Governance”, en Journal of Finance, vol. 52, no. 2, junio, pp. 737-783.

SURIÑACH, J., R. MORENO y R. ORTEGA-ARGILÉS, (2005): “Innovación y estructura de propiedad de las empresas españolas", en Colección Pensamiento PYME, Universidad de Barcelona, no. 2, agosto, pp. 9-65.

WANG, D., (2006): "Founding Family Ownership and Earnings Quality”, en Journal of Accounting Research, Vol. 44, No. 3, November, pp. 619.

WARFIELD, T. D., J. J. WILD y K. L. WILD, (1995): "Managerial Ownership, Accounting Choices, and Informativeness of Earnings", en Journal of Accounting and Economics, vol. 16, no. 1, julio, pp. 61-91.

YERMARCK, D., (1996): "Higher Market Valuation of Companies with a Small Board of Directors", en Journal of Financial Economics, vol. 40, no.2, febrero, pp. 185-211. 